\title{
Temperature-dependent hole detrapping for unprimed polycrystalline chemical vapor deposited diamond
}

\author{
Sigen Wang, ${ }^{\text {a) }}$ Paul Sellin, and Annika Lohstroh \\ Department of Physics, School of Electronics and Physical Sciences, University of Surrey, \\ Guildford GU2 7XH, United Kingdom
}

(Received 18 August 2005; accepted 15 November 2005; published online 9 January 2006)

\begin{abstract}
Rise-time distribution spectra of a polycrystalline chemical vapor deposited diamond detector were directly measured from alpha-particle induced pulse shapes over a temperature range of 240-280 K. Pulses due to hole-dominated charge transport showed a strong delayed component due to thermal detrapping of charge from a shallow level, with a mean rise time that decreased strongly with increasing temperature. The activation energy of this shallow hole trap was directly measured using an Arrhenius plot, with a value of $0.31 \pm 0.03 \mathrm{eV}$. No priming or pre-irradiation of the device was required in order to observe thermal detrapping, indicating that the concentration of shallow hole traps in this sample is relatively high. In contrast, no delayed component was observed from electron transport, indicating that only deep electron-trapping levels are active. (C) 2006 American Institute of Physics. [DOI: 10.1063/1.2162673]
\end{abstract}

Polycrystalline diamond grown by chemical vapor deposition (CVD) has been studied extensively for use as charged particle detectors operating under harsh radiation and thermal environments. This is due to diamond's unique mechanical and electrical properties, which include a wide band gap $(\sim 5.5 \mathrm{eV})$, high electron and hole mobilities (1800 and $1200 \mathrm{~cm}^{2} \mathrm{~V}^{-1} \mathrm{~s}^{-1}$, respectively), high resistivity $\left(>10^{11} \Omega \mathrm{cm}\right)$, and very low device dark currents. ${ }^{1,2}$ Unfortunately, the presence of defects and polycrystalline grain boundaries leads to significant charge trapping and recombination, resulting in reduced charge drift lengths and degraded detector performance. Various authors have tentatively identified trapping states in CVD diamond, although there is no clear picture of the role of particular levels, or bands of states, in affecting charge transport performance. However, it is accepted that CVD diamond contains both deep levels, responsible for short carrier lifetimes, and one or more shallow levels that are partially ionized at room temperature. Several techniques have been used to study the defect states in diamond; for example, Glesener ${ }^{3}$ measured a boronrelated level with an activation energy of $0.29 \mathrm{eV}$ in borondoped diamond using photoinduced current transient spectroscopy (PICTS). A wide range of deeper levels has been reported, including Gonon et al. ${ }^{4}$ and Hearne et al., ${ }^{5}$ who reported activation energies of 1.86 and $1.1 \mathrm{eV}$, respectively, measured using thermally stimulated current technique (TSC). Bruzzi et al. ${ }^{6}$ reported a variety of defect levels in electronic-grade polycrystalline CVD diamond, with activation energies in the range $0.3-1.5 \mathrm{eV}$, observed using both TSC and PICTS. Alpha particle response measurements are a powerful technique to separate the charge transport and carrier lifetimes of electrons and holes. Marinelli et $\mathrm{al}^{7}{ }^{7}$ used an analysis of alpha-particle pulse shapes as a function of temperature to deduce a "shallow" hole trap with an activation energy of $0.35 \mathrm{eV}$. In this letter, we report a new measurement of shallow trap states in CVD diamond, calculated from direct observation of the thermal trap emission rate

${ }^{a}$ Author to whom correspondence should be addressed; electronic mail: S.Wang@surrey.ac.uk or sigen_wang@yahoo.co.uk from alpha-particle pulse shapes. By digitally processing the preamplifier pulse shapes, pulse rise-time spectra are generated as a function of temperature, and an Arrhenius plot is deduced. An activation energy of $0.31 \pm 0.03 \mathrm{eV}$ for a shallow hole trap is determined. Unlike optical transient spectroscopy methods, such as PICTS, analysis of alpha-particle pulse shapes allows direct sampling of those trapping states that limit charge transport in diamond radiation detectors. The technique directly samples levels with a thermal emission rate in the range of $5 \times 10^{3}$ to $5 \times 10^{6} \mathrm{~s}^{-1}$. The nature of the state, either acting as an electron or hole trap, can also be directly inferred from the bias polarity of the sample. Consequently, this technique provides a valuable insight into the carrier trapping-detrapping processes in CVD diamond, and the role of these processes in defining the performance of polycrystalline diamond particle detectors.

The detector used in these measurements was fabricated from a $50 \mu \mathrm{m}$ thick unpolished free-standing CVD polycrystalline diamond film, with an average grain size on the growth surface of around $18 \mu \mathrm{m}$. The phase purity of the diamond was assessed by Raman spectroscopy, using a Renishaw micro-Raman spectrometer with an excitation source of a diode laser (782 nm line). Figure 1 shows a roomtemperature Raman spectrum of the sample, which shows only one sharp diamond characteristic peak at $1331.9 \mathrm{~cm}^{-1}$ with a full width at half-maximum of $5.3 \mathrm{~cm}^{-1}$. No other $s p^{2}$-bonded carbon phase peaks were detected, indicating a pure diamond phase. ${ }^{8,9}$

A detector structure was fabricated using large-area circular metal contact electrodes on both sides of the diamond film, forming a metal-semiconductor-metal sandwich structure. The diameter of the contacts was $6 \mathrm{~mm}$. No preirradiation was carried out on our device before the alphaparticle characterization. During the measurements the detector was placed on a temperature-controlled stage inside a vacuum chamber and irradiated using alpha particles from an uncollimated $5.49 \mathrm{MeV}^{241} \mathrm{Am}$ source, over a temperature range of $240-280 \mathrm{~K}$. The range of the alpha particles into the diamond was around $15 \mu \mathrm{m}$, as calculated using SRIM. ${ }^{10}$ The top contact of the detector, corresponding to the growth 


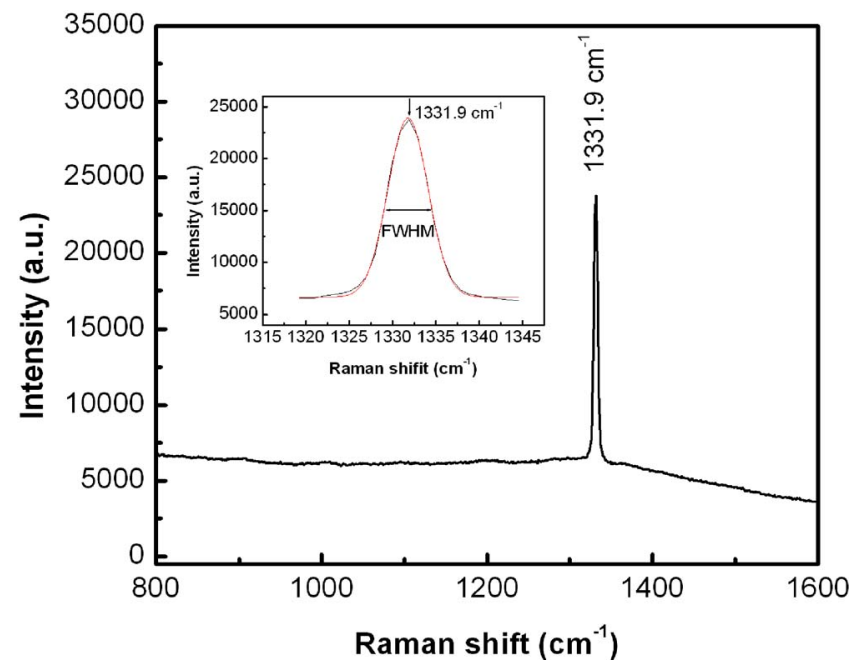

FIG. 1. (Color online) Room-temperature Raman spectrum of the CVD diamond film. Only a single peak at $1331.9 \mathrm{~cm}^{-1}$ was detected, characteristic of the diamond phase. Inset: the enlargement of the diamond characteristic peak.

surface of the diamond, was connected to a charge-sensitive preamplifier (Ortec model 142A), through which the bias voltage was applied. In these measurements a bias voltage of either +250 or $-250 \mathrm{~V}$ (equivalent to a field strength of $50 \mathrm{kV} / \mathrm{cm}$ ) was used in order to study the effect of either hole-dominated or electron-dominated charge transport, respectively. The preamplifier output was directly connected to a digital oscilloscope, which captured each pulse on an event-by-event basis. The preamplifier output was also connected to a multichannel analyzer (MCA) via a spectroscopy amplifier (Ortec model 570, shaping time of $2 \mu \mathrm{s}$ ), which acquired pulse height spectra.

Each alpha-particle interaction in the diamond detector creates $N_{0}$ free carriers of electrons and holes that drift apart a total distance $l$ under the action of the applied electric field, before either reaching the electrode or being trapped in a defect. The electron-hole pairs induce a total charge $Q$ in the external circuit that is given by $Q=e N_{0} l / d$, where $e$ is the electronic charge and $d$ is the detector thickness. Average carrier drift lengths in CVD diamond are typically in the range from $\sim 10$ to $>100 \mu \mathrm{m}$ depending on material quality, with a high density of trapping sites located at the crystallite boundaries.

In addition to the amplitude of the induced charge, analysis of the preamplifier pulse shapes can give important additional information, particularly related to thermal detrapping from shallow states in diamond. Deep trapping levels, for which there is no thermal re-emission over a measurable time scale, cause a curtailment of the prompt component of the preamplifier pulse rising edge. In contrast, the presence of shallow states that thermally re-emit over a $100 \mathrm{~ns}-100 \mu$ s time scale causes a slow "delayed" component to the preamplifier pulse rise time. Figures 2(a) and 2(b) show the preamplifier pulse shapes observed from our sample at bias voltages of $-250 \mathrm{~V}$ and $+250 \mathrm{~V}$. At $-250 \mathrm{~V}$, the pulse shapes due to electron transport contain only one prompt time component in the measured temperature range from 240 to $280 \mathrm{~K}$, as shown in Fig. 2(a). In contrast, at a bias of $+250 \mathrm{~V}$, the pulse shapes due to hole transport contain two time components to the rising edge; a prompt time component, and a slow component due to thermal emission Downloaded 30 Mar 2009 to 131.227 .178 .132 . Redistribution subje
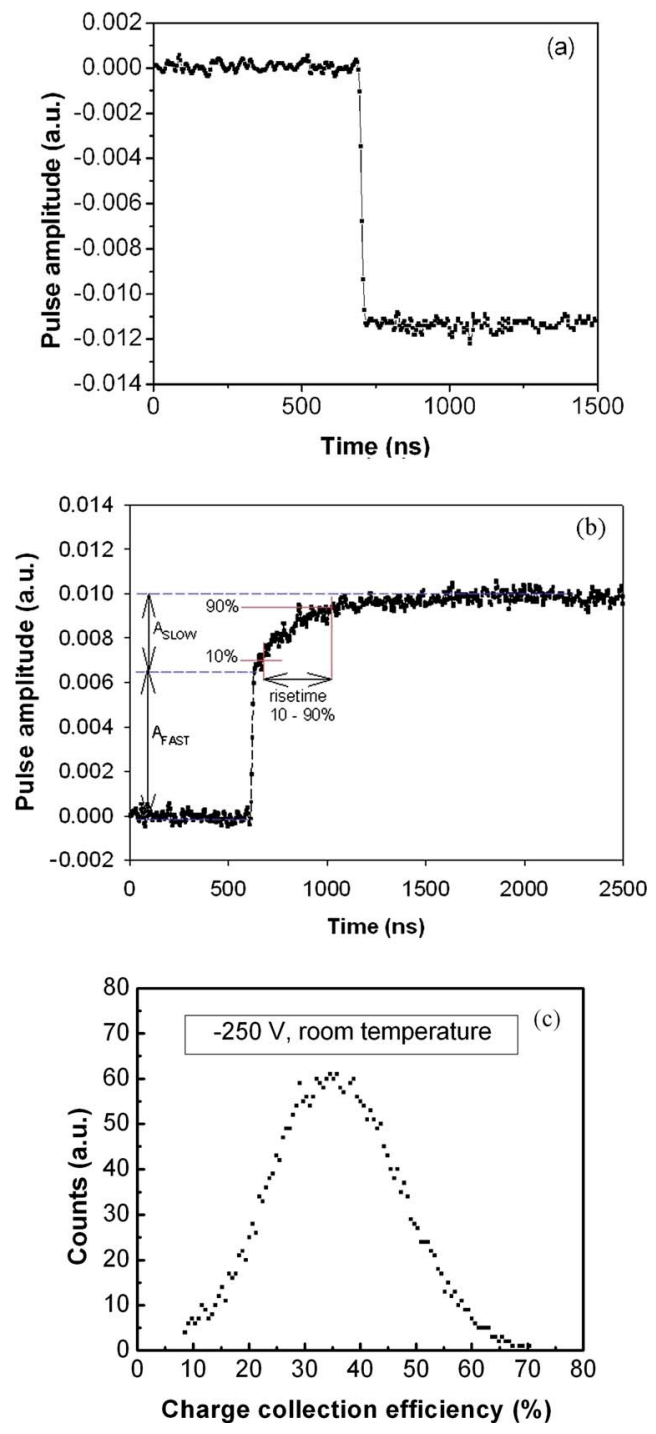

FIG. 2. (Color online) Typical alpha particle single pulse shapes obtained (a) at $-250 \mathrm{~V}$, (b) at $+250 \mathrm{~V}$, and (c) pulse height spectrum recorded at $-250 \mathrm{~V}$.

of trapped charge. A typical hole pulse shape is shown in Fig. 2(b), which indicates the amplitudes of the fast and slow components, $A_{\mathrm{FAST}}$ and $A_{\mathrm{SLOW}}$, respectively. Figure 2(c) shows the analog electron pulse height spectrum from the MCA, obtained at $-250 \mathrm{~V}$ at room temperature. A wellresolved alpha-particle peak was observed, comparable with that normally observed from good quality polycrystalline CVD diamond detectors. ${ }^{11,12}$ No resolvable alpha-particle peak was observed at a bias of $+250 \mathrm{~V}$ positive bias, indicating a substantially reduced mean drift length for holes.

For a bias of $-250 \mathrm{~V}$, the electron rise-time spectrum contained a well-resolved single peak with a centroid of $40 \mathrm{~ns}$ for all temperatures. This is considerably slower than the actual electron drift time in the CVD diamond sample (typically $<1 \mathrm{~ns}$ ), and is due only to the limiting rise time of the preamplifier. However, the absence of any rise-time component over time scales $>40 \mathrm{~ns}$ confirms that electron trapping is dominated by deep levels, which are too deep to exhibit measurable detrapping, as previously reported by Marinelli et al. ${ }^{12}$

For hole pulses, a significant slow component to the pulse shape was observed at a bias of $+250 \mathrm{~V}$. The $10 \%-$ $90 \%$ rise time of the slow component of each pulse was to AIP license or copyright; see http://apl.aip.org/apl/copyright.jsp 


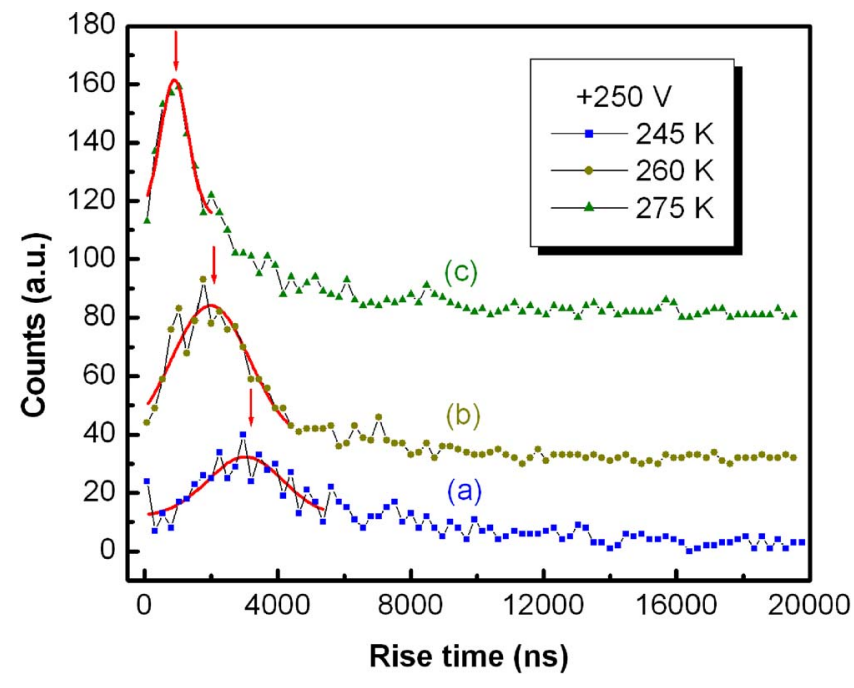

FIG. 3. (Color online) Typical rise-time distribution spectra observed at $+250 \mathrm{~V}$ at temperatures of (a) $245 \mathrm{~K}$, (b) $260 \mathrm{~K}$, and (c) $275 \mathrm{~K}$.

analyzed using LABVIEW software, and a rise-time histogram was acquired that contained a broad resolved peak. The centroid of the peak varied from several hundred nanoseconds to several microseconds over the temperature range 240-280 K, as shown in Fig. 3, providing direct evidence that the delayed component of the pulse shape is thermally activated. This phenomenon is ascribed to thermal reemission of holes from shallow trapping levels, which act in addition to hole trapping from deep levels where re-emission is not observed. Marinelli et al. ${ }^{12}$ also observed thermal reemission of holes from shallow traps in CVD diamond although in their device this was only seen after priming, or "pumping," of the diamond film by pre-irradiation with $\mathrm{x}$-rays that passivated the deep levels. The observation of detrapping from shallow states in our sample without the need for pre-irradiation suggests that the ratio of shallow/ deep traps is higher in our diamond film.

We also measured the rise-time distribution spectra at different positive biases, in the range +100 to $+400 \mathrm{~V}$, and found that there was no statistically significant change in the average rise time as a function of field strength. This confirms that the observed slow rise times are not due to direct (i.e., non-trap-assisted) hole transport.

The measured temperature-dependent rise times of the holes were analyzed in the form of an Arrhenius plot to obtain the activation energy of the shallow hole state, using a mean detrapping time $\tau$ calculated from the measured mean rise time, where $\tau=s_{0}^{-1} \exp \left(E_{A} / k T\right)$, and $s_{0}$ is the frequency or "escape" factor, $E_{A}$ is the activation energy of traps, $k$ is the Boltzmann constant, and $T$ is the temperature. The Arrhenius plot of $\ln (\tau)$ versus $1000 / T$ is shown in Fig. 4, with an activation energy deduced from the gradient of $E_{A}$ $=0.31 \pm 0.03 \mathrm{eV}$. The factor $s_{0}$, which measures the frequency at which the particle tries to exceed the barrier, was found to be $(3 \pm 5) \times 10^{-11} \mathrm{~Hz}$. The measured activation energy of $0.31 \mathrm{eV}$ of the state, or band of states, responsible for shallow hole trapping-detrapping, is close to that of $0.29 \mathrm{eV}$ measured by Glesener ${ }^{3}$ using photoinduced current transient spectroscopy in boron-doped diamond, and of $0.35 \mathrm{eV}$ calculated as the detrapping time by Marinelli et ll. $^{7}$ in undoped CVD diamond. The observed short hole mean drift length suggests that the charge carriers get

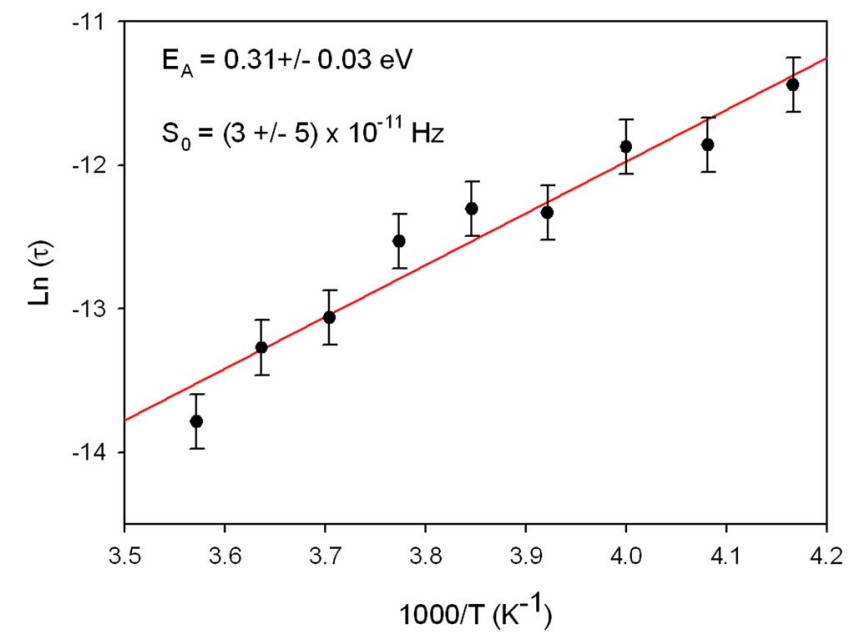

FIG. 4. (Color online) Arrhenius plot of $\ln (\tau)$ as a function of inverse temperature. The solid line is the best fit to the gradient.

trapped before they reach the grain boundaries, which is, therefore, consistent with the observed shallow level being located within the bulk of the diamond crystallites.

In conclusion, rise-time distribution spectra were directly measured at negative and positive bias of -250 and $+250 \mathrm{~V}$ over a temperature range from 240 to $280 \mathrm{~K}$, respectively. Only a prompt time component was observed from the electron-dominated pulse shapes, with a rise time of $40 \mathrm{~ns}$ due to the limiting preamplifier rise time. The lack of any slow component in the electron pulse shapes indicates that electrons are trapped at deep levels with no observable thermal detrapping. In contrast, significant delayed time components were observed in pulse shapes produced by hole transport when the detector was irradiated by alpha particles in the as-grown state. The strong reduction in mean detrapping time as a function of increasing temperature confirms the thermal origin of the slow time component, which is ascribed to thermal detrapping of holes from shallow levels acting as hole traps. The observation of thermal detrapping in our detector without pre-irradiation or priming suggests that the ratio of shallow/deep traps is high in our diamond film. An activation energy level of $0.31 \pm 0.03 \mathrm{eV}$ for the shallow hole state was directly determined from an Arrhenius plot derived from the temperature-dependent rise-time spectra.

${ }^{1}$ E.-K. Souw and R. J. Meilunas, Nucl. Instrum. Methods Phys. Res. A 400, 69 (1997).

${ }^{2}$ P. J. Sellin, M. B. H. Breese, A. P. Knights, L. C. Alves, R. S. Sussmann, and A. J. Whitehead, Appl. Phys. Lett. 77, 913 (2000).

${ }^{3}$ J. W. Glesener, Appl. Phys. Lett. 63, 767 (1993).

${ }^{4}$ P. Gonon, S. Prawer, and D. Jamieson, Appl. Phys. Lett. 70, 2996 (1997).

${ }^{5}$ S. M. Hearne, D. N. Jamieson, E. Trajkov, S. Prawer, and J. E. Butler, Appl. Phys. Lett. 84, 4493 (2004).

${ }^{6}$ M. Bruzzi, D. Menichelli, S. Sciortino, and L. Lombardi, J. Appl. Phys. 91, 5765 (2002).

${ }^{7}$ M. Marinelli, E. Milani, A. Paoletti, G. Pucella, A. Tucciarone, G. VeronaRinati, M. Angelone, and M. Pillon, Diamond Relat. Mater. 12, 1733 (2003).

${ }^{8}$ S. A. Solin and A. K. Ramdas, Phys. Rev. B 1, 1687 (1970).

${ }^{9}$ P. V. Huong, Diamond Relat. Mater. 1, 33 (1991).

${ }^{10}$ J. F. Ziegler, J. P. Biersack, and U. Littmark, The Stopping and Range of Ions in Solids (Pergamon, New York, 1985).

${ }^{11}$ A. Brambilla, D. Tromson, P. Bergonzo, C. Mer, and F. Foulon, IEEE Trans. Nucl. Sci. 49, 277 (2002).

${ }^{12}$ M. Marinelli, E. Milani, A. Paoletti, A. Tucciarone, G. Verona-Rinati, M. Angelone, and M. Pillon, Phys. Rev. B 64, 195205 (2001). 